\title{
A NEW SYNDROME OF MULTIPLE HEMANGIOMAS, RIGHT DOMINANT DOUBLE AORTIC ARCH, AND COARCTATION
}

C. H. Wong, MA, MB, BChir, FRCS, FRCSEd, J. G. Wright, MA, MB, BChir, FRCP, FRCPCH, E. D. Silove, FRCP,

R. Willetts, ACP, and W. J. Brawn, FRCS, Birmingham, United Kingdom

Congenital aortic lesions are occasionally associated with clinical syndromes. Recognition of these syndromes is important in aiding clinical diagnosis and accurate surgical management. We describe the case histories of 2 children who appear to have a new vascular syndrome comprising right dominant dou-

From the Department of Cardiac Surgery, Birmingham Children's Hospital, Birmingham, United Kingdom.

J Thorac Cardiovasc Surg 2001;121:1207-9

Copyright (C) 2001 by The American Association for Thoracic Surgery

0022-5223/2001 $\$ 35.00+0 \quad \mathbf{1 2 / 5 4 / 1 1 2 6 2 7}$

doi: $10.1067 / \mathrm{mtc} .2001 .112627$ ble aortic arch, type B interruption of the left arch, coarctation, aberrant subclavian arteries, and associated multiple right-sided facial and upper limb superficial and deep hemangiomas.

\section{Clinical summaries}

PATIENT 1. A baby girl was born by elective cesarean birth for cephalopelvic disproportion at term in August 1984 and required ventilatory assistance for 6 days for hyaline membrane disease. During this period she was found to have a continuous cardiac murmur and heart failure. There was no history of congenital heart disease in the family, which included one healthy sibling. Multiple capillary hemangiomas were noted over the right side of her face, shoulder, and arm and a deeper hemangioma was present on the back of her neck. 


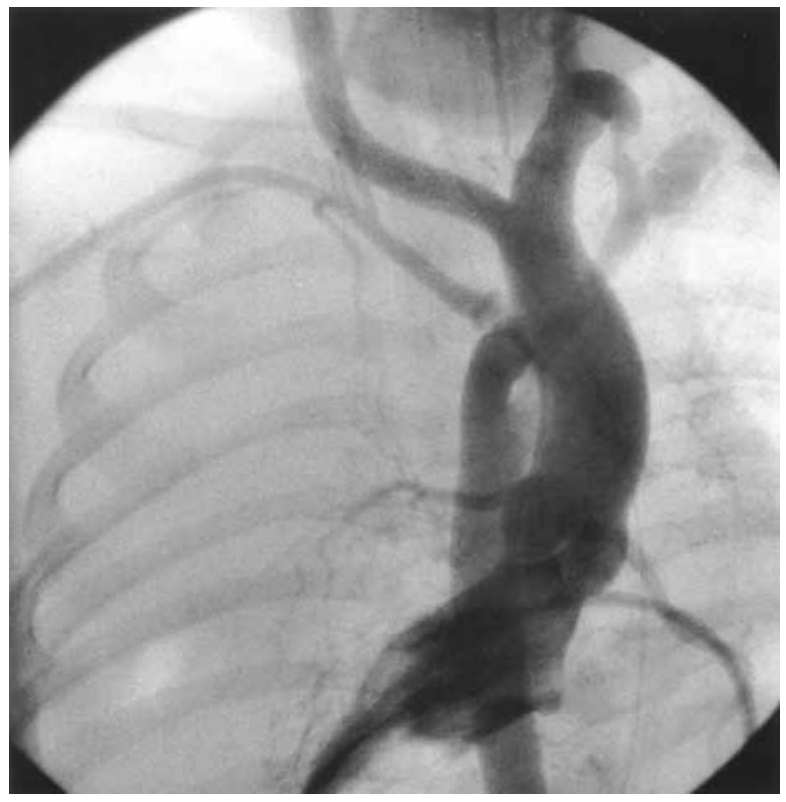

Fig 1. Aortogram demonstrating aberrant left subclavian artery causing tracheal compression.

Cardiac catheterization demonstrated a high left ventricular pressure of $200 \mathrm{~mm} \mathrm{Hg}$, a right aortic arch with an anomalous left subclavian arising from the descending aorta, and coarctation distal to their origins. There were no intracardiac abnormalities.

The infant underwent elective repair of the coarctation on October 18, 1984, at the age of 2 months. The operation was performed through a thoracotomy in the left fourth intercostal space. The ductus arteriosus was divided, the aorta was mobilized, the coarctation resected, and the descending aorta was anastomosed to the side of the ascending aorta.

She returned to the intensive care unit in stable condition, but she remained dependent on mechanical ventilation, with marked head retraction each time she was extubated. Tracheal compression by a vascular ring was suspected, and further investigations with a barium swallow demonstrated a posterior indentation of the esophagus. A second aortogram demonstrated the left aberrant subclavian artery traveling to the trachea associated with a left aortic arch with a type B interruption (Fig 1).

November 21, 1984, she underwent a second left thoracotomy and division of the posterior segment of the vascular ring between ligatures. After the procedure, she was quickly extubated and recovered rapidly. She has since been examined regularly in the clinic and had a repeat aortogram after 7 years. No recurrence of the coarctation was found and no gradient was detected across the anastomosis. Currently, she remains well with no evidence of neurologic or ophthalmic lesions.

PATIENT 2. A baby boy was born at term in January 2000. He had no apparent problems at postnatal examination and was feeding well. However, at 2 months he had an episode of high-pitched crying. His breathing was shallow and he became unresponsive, cold, pale, and clammy.
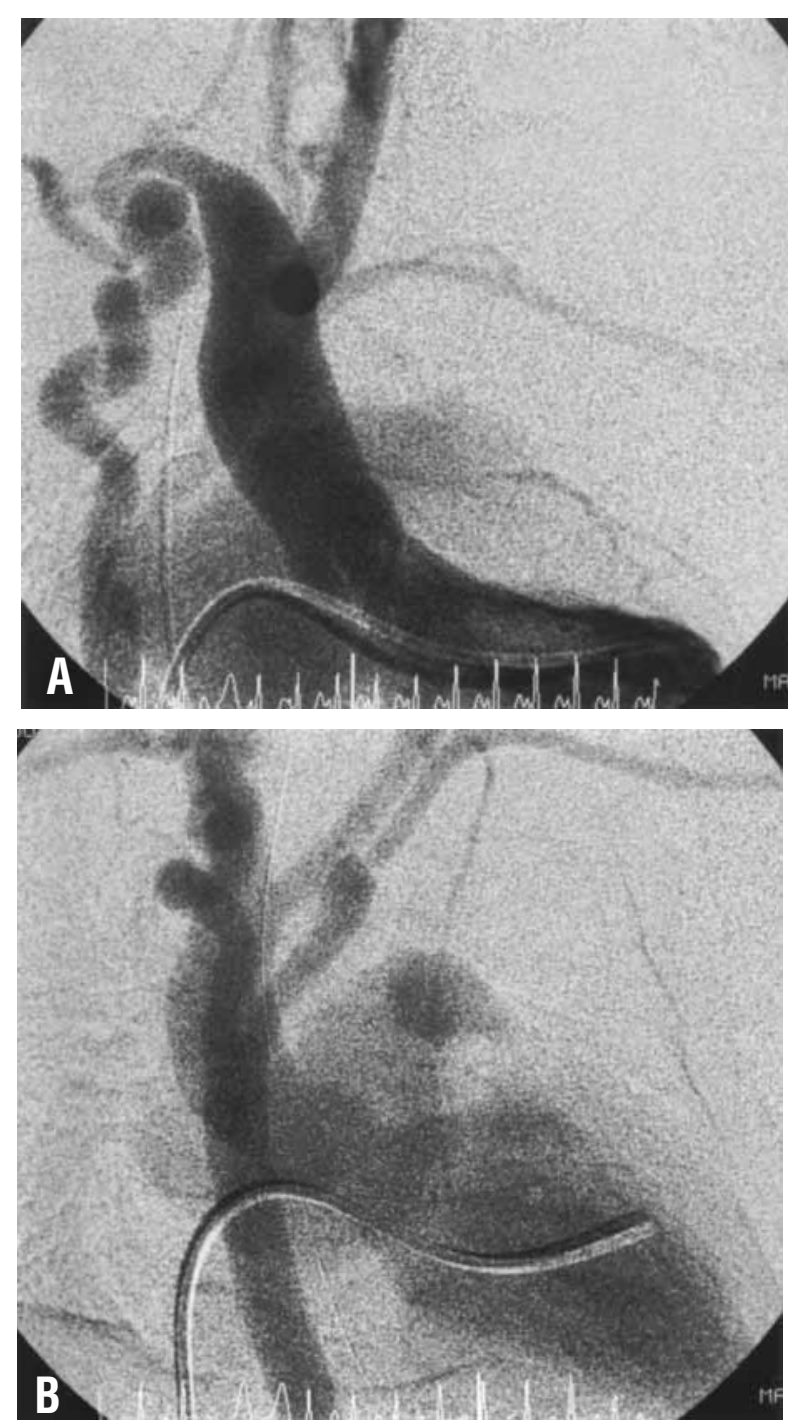

Fig 2. Aortogram demonstrating the right aortic arch, aberrant subclavian artery, and tortuous coarctation segment.

On admission to the hospital, examination demonstrated a pansystolic cardiac murmur, a $20-\mathrm{mm} \mathrm{Hg}$ difference in mean blood pressure between the upper and lower limbs, and absent femoral pulses. There was no family history of congenital heart disease or other inherited conditions. He had right-sided capillary hemangiomas on his face and a deep hemangioma of the right eye that was causing unilateral exophthalmos.

Catheterization demonstrated a double aortic arch with a right dominant arch and type B interruption of the left arch. The aorta acutely narrows after the right head and neck vessels and forms a long tortuous psuedocoarctation segment before joining the descending aorta. The left subclavian artery arose from the descending aorta (Fig 2). No intracardiac abnormalities were found on catheterization or echocardiography.

Elective repair was performed through a median sternotomy. Cardiopulmonary bypass with hypothermic circulatory 
arrest at a $15^{\circ} \mathrm{C}$ nasopharyngeal temperature was performed while the ascending arch, arch, and descending aorta were fully mobilized. The long tortuous left subclavian artery was disconnected in the superior mediastinum and anastomosed end to side to the left carotid artery. Cold crystalloid cardioplegic solution was administered and profound hypothermic circulatory arrest used to repair the long coarctation.

The aorta was divided distal to the right subclavian artery. This left a fork comprising of the tortuous collateral segment, a remnant of the posterior left aortic arch, and the aberrant left subclavian artery. The fork was opened along the common inner surface and sutured to form a single tube approximately $10 \mathrm{~mm}$ in diameter. The proximal end of the neoaorta was anastomosed to the side of the ascending aorta. Total bypass time was 107 minutes with 20 minutes of circulatory arrest.

The boy recovered without complications, and computed tomographic scans of the head were performed postoperatively to exclude PHACE syndrome. ${ }^{1}$ No cerebellar lesions were found and the eyes were normal.

Discussion. Capillary hemangiomas (strawberry nevi) are common vascular lesions, occurring in $8 \%$ of all neonates, and are usually solitary $(90 \%)$. However, the presence of multiple hemangiomas is associated with several syndromes. ${ }^{2}$ In these conditions, the cutaneous capillary hemangiomas are flatter and may occur in unusual locations in deep tissue.

Coarctation is one of the more common congenital cardiovascular lesions, comprising $5 \%$ to $8 \%$ of all congenital heart lesions, but right aortic arch and double aortic arches are rare. These lesions occur as a result of developmental abnormalities in the embryo ${ }^{3}$ but can in exceptional cases be transmitted as an autosomal dominant trait. ${ }^{4}$

A total of 2 children have been previously described with the association of capillary hemangiomas, right aortic arch, and coarctation, ${ }^{5,6}$ with 2 other children reported with right aortic arch and coarctation having associated cavernous hemangiomas. ${ }^{7}$ However, none of these reported cases had double aortic arches. Additionally, in both of our patients there was a type B interruption of the left arch. The failure to recognize this in the first child at the time of operation necessitated a second procedure to relieve tracheal compression.

The PHACE syndrome is a neurocutaneous syndrome that consists of Posterior fossa malformations, Hemangiomas, Arterial anomalies, Coarctation of the aorta and cardiac defects, and Eye abnormalities. ${ }^{1}$ The lack of neurologic, ophthalmic, and cardiac abnormalities and the remarkably similar findings in our cases suggest that they may form a new clinical syndrome consisting of vascular lesions only.
An important observation in these children is that the cutaneous hemangiomas occur only on the right side on the face and upper limb, with at least one deep hemangioma in each case in an unusual location. Thus, there is a strong possibility of an underlying genetic lesion in the etiology in addition to environmental factors during embryologic development. Identification of such a locus may help our understanding of the etiology of coarctation and congenital lesions of the aorta. As the first case demonstrated, recognition of this syndrome is important in a full diagnosis and operative planning.

Received for publication Oct 24, 2000; accepted for publication Oct 31, 2000.

Address for reprints: Carl Wong, MA, MB, BChir, FRCS, FRCSEd, Specialist Registrar, Department of Cardiac Surgery, Freeman's Hospital, High Heaton, Newcastle NE7 7DN, United Kingdom (E-mail: wongch@ postmaster.co.uk).

\section{REFERENCES}

1. Frieden IJ, Reese V, Cohen D. PHACE syndrome: the association of posterior fossa brain malformations, hemangiomas, arterial anomalies, coarctation of the aorta and cardiac defects, and eye abnormalities. Arch Dermatol 1996;132:307-11.

2. Burns AJ, Kaplan LC, Mulliken JB. Is there an association between hemangioma and syndromes with dysmorphic features? Pediatrics 1991;88:1257-67.

3. Tikkanen J, Heinonen OP. Risk factors for coarctation of the aorta. Teratology 1993;47:565-72.

4. Gerboni S, Sabatino G, Mingarelli R, Dallapiccola B. Coarctation of the aorta, interrupted aortic arch, and hypoplastic left heart syndrome in three generations. J Med Genet 1993;30:328-9.

5. Honey M, Lincoln JC, Osborne MP, de Bono DP. Coarctation of aorta with right aortic arch: report of surgical correction in 2 cases: one with associated anomalous origin of left circumflex coronary artery from the right pulmonary artery. Br Heart $\mathbf{J}$ 1975;37:937-45

6. Vaillant L, Lorette G, Chantepie A, Marchand M, Alison D, Vaillant MC, et al. Multiple cutaneous hemangiomas and coarctation of the aorta with right aortic arch. Pediatrics 1988;81:70710.

7. Schneeweiss A, Blieden LC, Shem-Tov A, Motro M, Feigel A, Neufeld HN. Coarctation of the aorta with congenital hemangioma of the face and neck and aneurysm or dilatation of a subclavian or innominate artery: A new syndrome? Chest 1982;82:186-7. 